\title{
SParticle, an algorithm for the analysis of filamentous microorganisms in submerged cultures
}

\author{
Joost Willemse $\cdot$ Ferhat Büke $\cdot$ Dino van Dissel $\cdot$ Sanne Grevink • \\ Dennis Claessen • Gilles P. van Wezel
}

Received: 5 July 2017/ Accepted: 5 September 2017/Published online: 15 September 2017

(C) The Author(s) 2017. This article is an open access publication

\begin{abstract}
Streptomycetes are filamentous bacteria that produce a plethora of bioactive natural products and industrial enzymes. Their mycelial lifestyle typically results in high heterogeneity in bioreactors, with morphologies ranging from fragments and open mycelial mats to dense pellets. There is a strong correlation between morphology and production in submerged cultures, with small and open mycelia favouring enzyme production, while most antibiotics are produced mainly in pellets. Here we describe SParticle, a Streptomyces Particle analysis method that combines whole slide imaging with automated image analysis to characterize the morphology of submerged grown Streptomyces cultures. SParticle allows the analysis of over a thousand particles per hour, offering a high throughput method for the imaging and statistical analysis of mycelial morphologies. The software is available as a plugin for the open source software ImageJ and allows users to create custom filters for other microbes. Therefore, SParticle is a
\end{abstract}

Electronic supplementary material The online version of this article (doi:10.1007/s10482-017-0939-y) contains supplementary material, which is available to authorized users.

J. Willemse - F. Büke - D. van Dissel .

S. Grevink · D. Claessen · G. P. van Wezel $(\bowtie)$

Molecular Biotechnology, Institute of Biology, Leiden

University, Sylviusweg 72, 2333 BE Leiden,

The Netherlands

e-mail: g.wezel@biology.leidenuniv.nl widely applicable tool for the analysis of filamentous microorganisms in submerged cultures.

Keywords Image analysis · Morphology ·

Filamentous microorganisms $\cdot$ Actinobacteria $\cdot$ Whole slide imaging $\cdot$ Image J

$\begin{array}{ll}\text { Abbreviations } \\ \text { AR } & \text { Aspect ratio } \\ \text { B\&W } & \text { Black \& white } \\ \text { Cfu } & \text { Colony forming units } \\ \text { COPAS } & \begin{array}{l}\text { Complex object parametric analyzer and } \\ \text { sorter }\end{array} \\ \text { Gb } & \text { Gigabyte } \\ \text { MS } & \text { Mannitol soy flour agar } \\ \text { RAM } & \text { Random access memory } \\ \text { ROI } & \text { Region of interest } \\ \text { Rpm } & \text { Rotations per minute } \\ \text { TIFF } & \text { Tag image file format } \\ \text { TSBS } & \text { Tryptic soy broth with sucrose } \\ \text { WSI } & \text { Whole slide imaging }\end{array}$

\section{Introduction}

Streptomycetes are Gram-positive bacteria that have a complex mycelial lifestyle, whereby they reproduce via sporulation (Claessen et al. 2014; Chater and Losick 1997). Streptomycetes and other members of the phylum Actinobacteria are of great medical, 
biotechnological and agricultural importance due to their ability to produce a wide variety of bioactive natural products (Barka et al. 2016; Bérdy 2005; Hopwood 2007). Furthermore, their saprophytic lifestyle allows them to degrade almost all known, natural bio-polymers (Omura 1992). Morphology and growth characteristics may vary strongly, based on strainspecific traits that are yet poorly understood (Jakimowicz and van Wezel 2012). During growth in submerged cultures, Streptomyces species form mycelial structures, which can be classified in three groups based on the different phenotypes (van Dissel et al. 2014). Many streptomycetes form extended mycelial networks, which often self-aggregate into dense cellular structures called pellets (Celler et al. 2012; Meyerhoff et al. 1995). Other morphologies include mycelial mats (caused by open growth) and small hyphal fragments (Tresner et al. 1967). Streptomyces can be further subdivided into those that sporulate in liquid cultures and those that do not (Girard et al. 2013; Glazebrook et al. 1990; Kendrick and Ensign 1983). When grown under different conditions, growth rate and morphology are not just strain specific, but also change depending on the composition of the growth medium, $\mathrm{pH}$, temperature, shear rate, dissolved oxygen concentration and inoculum (Tough and Prosser 1996; Cui et al. 1998). The relationship between growth and morphology, on the one hand, and biomass accumulation and productivity on the other, is complicated, and optimal morphology varies from product to product (van Wezel et al. 2009). While pellets are primarily physiologically active around the edges and therefore associated with slow growth, they promote antibiotic production, presumably correlated to nutrient limitation in the core of the pellets (van Dissel et al. 2014; Wardell et al. 2002). Conversely, smaller pellets and open mycelia are generally preferred morphologies for enzyme production, which may be explained by the fact that protein production preferentially takes place at apical sites (van Wezel et al. 2006; Willemse et al. 2012).

Detailed assessment of the morphological characteristics of a culture is important for rational strain design (Celler et al. 2012). Attempts in the 1990s aimed at qualitatively assessing the morphology of filamentous microorganisms, applied a combination of microscopy with the advances in computational power for (semi-)automated image analysis to measure the size and other characteristics such as shape, density and complexity of pellets (Reichl et al. 1992; Reichl et al. 1990; Treskatis et al. 1997; Pinto et al. 2004; Barry and Williams 2011). A limitation of microscopy-based techniques is the trade-off between the throughput and image quality. Sufficient resolution is required to allow reliable image analysis, but higher magnification limits the number of particles that can be captured (Barry and Williams 2011; Papagianni 2014; Hardy et al. 2017). Various methods have been tried to increase the sample size in image analysis methods (Cox and Thomas 1992; Packer and Thomas 1990). Flow cytometry using a complex object parametric analyzer and sorter (COPAS) efficiently analyzes pellets by size and fluorescence, allowing up to several $100 \mathrm{~s}$ of measurements per second (Petrus et al. 2014). There are however technical limitations; firstly, detection of small mycelial fragments is unreliable because their extinction coefficient is close to the background value, and the same is true for hyphae at the edge of open mycelial aggregates, making the relation between time of flight and pellet size hard to calibrate. Secondly, the time of flight fails to take the shape of pellets into account. Since pellets are typically non-circular, COPAS measurements cannot be used for pellet-volume estimation. This is also reflected in the larger standard deviation often observed in cytometry measurements (Rønnest et al. 2012). Thus, microscopy allows acquiring information about pellet shape, density and fragmentation, while flow cytometry is ideal for studying population traits, due to the high number of measurements.

To overcome the low sample size in microscopic measurements we applied whole slide imaging (WSI) combined with automated image analysis to increase the throughput without compromising image quality and thus reliability. WSI has been used by pathologists for more than a decade (Webster and Dunstan 2014). Imaging of a whole pathological sample and subsequent automated image analysis is becoming common practice in areas such as cancer research (Cooper et al. 2015) and developments on staining techniques continuously expand the applicability of WSI (Gray et al. 2015). So far, WSI has found limited application in microbial population measurements (Posch et al. 2012), and no software is currently freely available. Here we describe a WSI-based method for automated imaging of Streptomyces mycelia. By capturing a high-resolution picture of a large area, our approach visualizes a large number of particles, and accurately 
identifies particles ranging from small mycelial fragments to large pellets. As our method is applicable to other filamentous micro-organisms, we expect that this free software will allow labs to improve the quantitatively and qualitatively description of microbial morphologies.

\section{Materials and methods}

Strains and culturing conditions

Streptomyces coelicolor FM145 (Willemse and van Wezel 2009) was used as reference strain in all experiments. FM145 is a derivative of $S$. coelicolor M145 (Bentley et al. 2002) with reduced autofluorescence. The filamentous fungus Aspergillus niger N402, as well as the cyanobacteria Gloeothece sp. PCC 6909, Chroococcidiopsis sp. PCC 6712 and Synechococcus elongatus PCC 7942 were obtained from our in-house strain collection. A. niger N402 was grown at $30{ }^{\circ} \mathrm{C}$ in complete medium, consisting of minimal medium supplemented with $1 \%$ yeast extract and $0.5 \%$ casamino acids (Vinck et al. 2005). Gloeothece sp. PCC 6909, Chroococcidiopsis sp. PCC 6712 and S. elongatus PCC 7942 were cultured in BG-11 media as described (Voshol et al. 2015). Streptomyces spores were collected from MS agar plates (Kieser et al. 2000). Colony forming units (Cfus) were determined by plating serial dilutions on MS agar plates. For submerged growth and pellet size measurement experiments, S. coelicolor was cultivated in spring-loaded $125 \mathrm{ml}$ Erlenmeyer flasks containing $25 \mathrm{ml}$ Tryptic Soy Broth media supplemented with $10.3 \%$ sucrose (TSBS). Cultures of $S$. coelicolor FM145 were inoculated with $10^{6} \mathrm{Cfu}$ spores $/ \mathrm{ml}$ unless stated otherwise. A one inch orbital shaker (New Brunswick) was used at $30{ }^{\circ} \mathrm{C}$ with shaking at $200 \mathrm{rpm}$.

Microscopy and software

For imaging of the samples, we used a setup consisting of a Zeiss inverted Axio Observer with automated XYstage using a $\times 10$ objective and a Hamamatsu EMCCD c9100-02 camera. Samples were taken from submerged cultures using a wide-gauge $200 \mu$ l pipette tip to allow big pellets to pass, and transferred to a microscope slide, covered with a $2 \times 2 \mathrm{~cm}^{2}$ cover slip and sealed by using fast drying nail polish to prevent evaporation induced artefacts during imaging. The automated stage served to capture a multi-image phase-contrast mosaic, imaged by meandering from top left to bottom-right (Fig. S1). The outer $2 \mathrm{~mm}$ of the cover slip were excluded from measurements to exclude an effect of the sealing material. By using a 20 by 20 grid, with $10 \%$ overlap of images, we were able to capture the $\sim 1.4 \times 1.4 \mathrm{~cm}$ inner area of the slide, which takes $\sim 8$ min to complete. The software was developed as a plugin for the ImageJ package (https:// imagej.nih.gov/ij/index.html), which is freely available from the Leiden University ImageJ update site (https://imagej.net/List_of_update_sites).

\section{Results}

Automated image analysis steps

We sought to develop software that allows researchers to reliably assess morphological parameters of submerged cultures of filamentous microorganisms at medium to high throughput scale. A major aim was to combine automated image analysis with automated whole slide imaging so as to allow assessing the morphology of mycelia in submerged cultures for any given culture within $15 \mathrm{~min}$ in a statistically relevant manner.

\section{Mosaic image building and image normalization}

Submerged cultures were set up by inoculating TSBS cultures with $10^{6} \mathrm{cfu}$ and grown for $24 \mathrm{~h}$ at $30{ }^{\circ} \mathrm{C}$. Subsequently, mosaic images of the samples were obtained using an automated stage microscope. To quickly analyze a large number of mycelia, mosaic images were created using the Axiovision software and stored as separate TIFF files. These files were reconstructed into large mosaic images thereby eliminating partial pellets at the borders of individual images. Furthermore, the images were normalized to correct for light fluctuations from the light source. Using a $20 \times 20$ grid, with $10 \%$ overlap of images, an approximately $1.4 \times 1.4 \mathrm{~cm}$ inner area of the slide was captured in approximately $8 \mathrm{~min} .400$ images per slide were taken and analyzed in ImageJ. As a rule of thumb, $12 \mathrm{~Gb}$ of RAM is sufficient to analyze $\sim 325$ megapixel images, which means that an entire 
microscope slide can be analyzed as a single image. Sampling density was chosen such that most pellets and fragments are spatially separated, simplifying analysis of the objects and allowing brightness adjustment of each mosaic image using the background intensity determined as grey values. This method also allows analysis of fluorescence images, as well as analysis of different mycelial morphologies (Zacchetti et al. 2016).

\section{Determining the region of interest $(\mathrm{ROI})$}

Thresholding was applied to separate candidate objects from the background, creating a binary image. Pixels with brightness values between 50 and 90 were assigned as background, while the remaining pixels were assigned as possible pellet edges and small mycelial fragments. The detection of small fragments was facilitated by phase-contrast imaging, where changes in light permeability are emphasized resulting in illuminated edges. After thresholding, area filling was applied to generate solid objects out of the detected edges (Fig. 1). Objects larger than $6 \times 10^{5} \mu \mathrm{m}^{2}$ (corresponding to an object with a diameter larger than $900 \mu \mathrm{m}$ ) were ignored to remove artifacts such as air bubbles, while objects smaller than 200 pixels were filtered out to remove small artifacts like cell debris or precipitated salts.

To maximize sample size and optimize the accuracy of the measurements, groups of particles were separated by water-shedding the mask after applying a Gaussian blur. This approach separates adjacent objects as shown in Fig. 1e. The risk of separating irregularly shaped pellets into two objects is minimized by only applying a separation if the interface between the two adjacent particles is smaller than $150 \mu \mathrm{m}$. Separation between adjacent objects is achieved by using a Voronoi transformation. This transformation divides the image into as many regions as there are objects in the total image (Fig. 1).

A rule set was applied on the ROIs to separate contaminants from pellets or mycelial fragments. For example, this filtering step eliminates large noncircular objects (e.g. cotton fibers) and objects deviating from the normal pellet density. For a complete set of rules, we refer to the Supplemental Methods. To allow a broader use of the plugin, a manually defined filter can be applied to any given set of ROIs. An additional macro tool is provided to create these manual filters. In this paper only the settings,

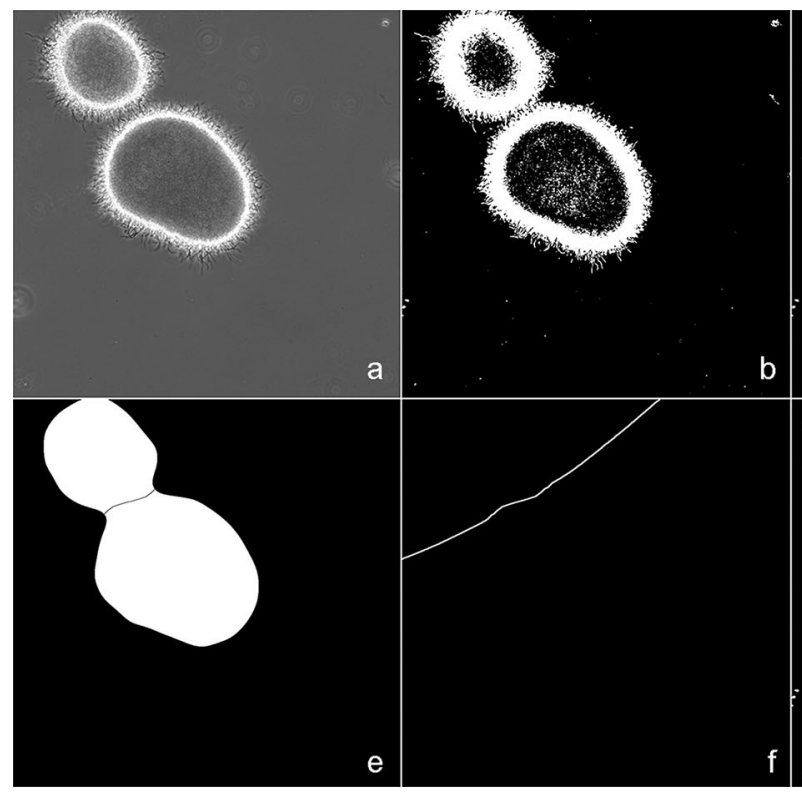

Fig. 1 Steps of ROI identification. The original image (a) is transformed into black \& white $(\mathrm{B} \& \mathrm{~W} ; \mathbf{b})$ using thresholding. Fully enclosed black areas are filled with white (c) and a Gaussian blur (d) is applied. Areas that are connected via "thin" bridges are separated via Watershedding (e) and a Voronoi

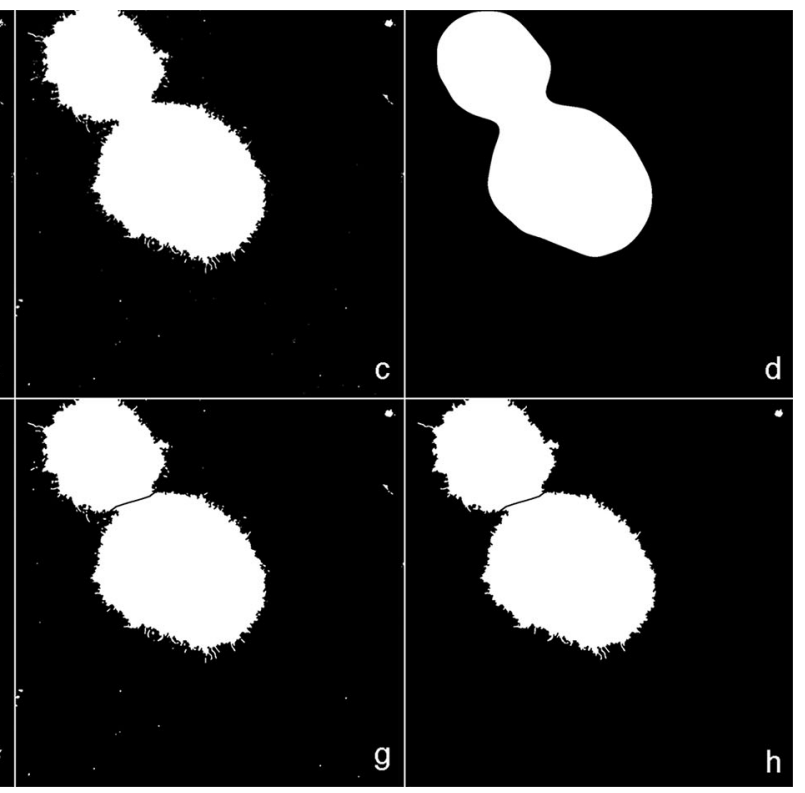

transformation (f) is applied to find object areas. Identified area borders are subtracted from the filled B\&W image (g) and small objects are deleted (h). Individual areas are then marked as ROIs, which are now ready for advanced filtering to further analyse the particles 
optimized for streptomycetes, were used (see Supplemental Methods). Depending on the density of the sample, an image may contain up to 500 pellets and mycelial fragments. These are all identified and marked as separate ROIs and further measured. Saving all regions as well as the selected ones in separate files allows manual correction for false negatives if required.

\section{Determining particle shape}

After eliminating non-desirable objects from the potential list of particles, multiple parameters need to be measured to describe the objects in more detail. We chose the following parameters to describe the objects of interest: area, mean intensity, standard deviation of intensity, roundness, circularity and Feret's diameter (an object's diameter along its longest axis). In ImageJ, the area to be measured is rotated with $2^{\circ}$ increments and Feret's diameter is calculated vertically. The maximum measurement is taken as the longest distance within the object.

After processing by ImageJ, all selected ROIs were subjected to additional measurements. Initially the roughness was determined by dividing the perimeter of the object by the perimeter of the fitted ellipse. Furthermore, the morphological number, a dimensionless parameter which combines multiple morphological parameters and has been shown to correlate to productivity, was determined (Wucherpfennig et al. 2011; Wucherpfennig et al. 2013), and subsequently the fractal box mass dimension as well as the box surface dimension and the fractal quotient are determined (Obert et al. 1990; Ryoo 1999). These three morphological pellet parameters distinguish different mycelial morphologies. Another circularity measurement based on Cartesian to Polar coordinate transformation (Stojmenovic et al. 2013) was also determined. This method is more suited for estimating the circularity of a pellet, as other conventional methods use the perimeter length of an object. The object perimeter of a mycelial particle is highly dependent on outwardly protruding hyphae. Therefore, the number and length of the hyphae dictates conventional circularity calculations, making them unsuited to describe the circularity (see Fig. S2 for more details). We also implemented a band-intensity measurement, where donut shaped areas are placed that radiate from the center of the shape. The shapes of the donuts are corresponding to the shape of the ROI and each ROI is divided into 20 regions. By default, pellet images in phase contrast microscopy have relatively dark protruding hyphae, then a brighter ring at which the pellets dense region starts, followed by a darker interior. We make use of this by determining the peak intensity of the selected bands and then determining its positions relative to the edge of the pellet. This determines the width of the less dense outer perimeter of the pellet. The difference between the edge and the maximum allows determining the size of the lowdensity exterior in micrometers. Finally, we determine the outer intensity ratio to the inner intensity to determine how dense the pellets are, this parameter can be helpful in assisting to differentiate between pellet (with a dense/dark center) and mat formers (open center).

From plugin to practice: imaging pellet formation by Streptomyces coelicolor

\section{Optimizing the software for imaging of Streptomyces mycelia}

Next, we optimized the microscope settings for the analysis of Streptomyces mycelia. A $\times 10$ magnification results in a pixel size of $780 \mathrm{~nm}$, which suffices to distinguish mycelial fragments larger than $2 \mu \mathrm{m}$, which we chose as cut-off. We used this magnification throughout all experiments to minimize capture time while maintaining sufficient resolution. 42 WSI images were analyzed, resulting in the identification of more than 10,000 mycelial objects. To check the efficiency of the filter we manually confirmed or rejected all identified objects. This revealed $1.5 \pm 0.46 \%$ false positives (average 262 correct objects per image detected, and 4 incorrect ones). Manual inspection of over 27,000 rejected objects revealed $1.06 \pm 0.02 \%$ false negatives, in other words rejected objects that are proper mycelial objects (on average 658 correctly rejected objects per image detected, and 7 falsely rejected).

To test the reproducibility of the particles imaged by WSI combined with the ImageJ plugin SParticle, $S$. coelicolor was cultivated using the standard conditions described above. From a single flask three separate samples were analyzed to detect measurement variations. Comparing morphological descriptive parameters, including area, Feret diameter and 

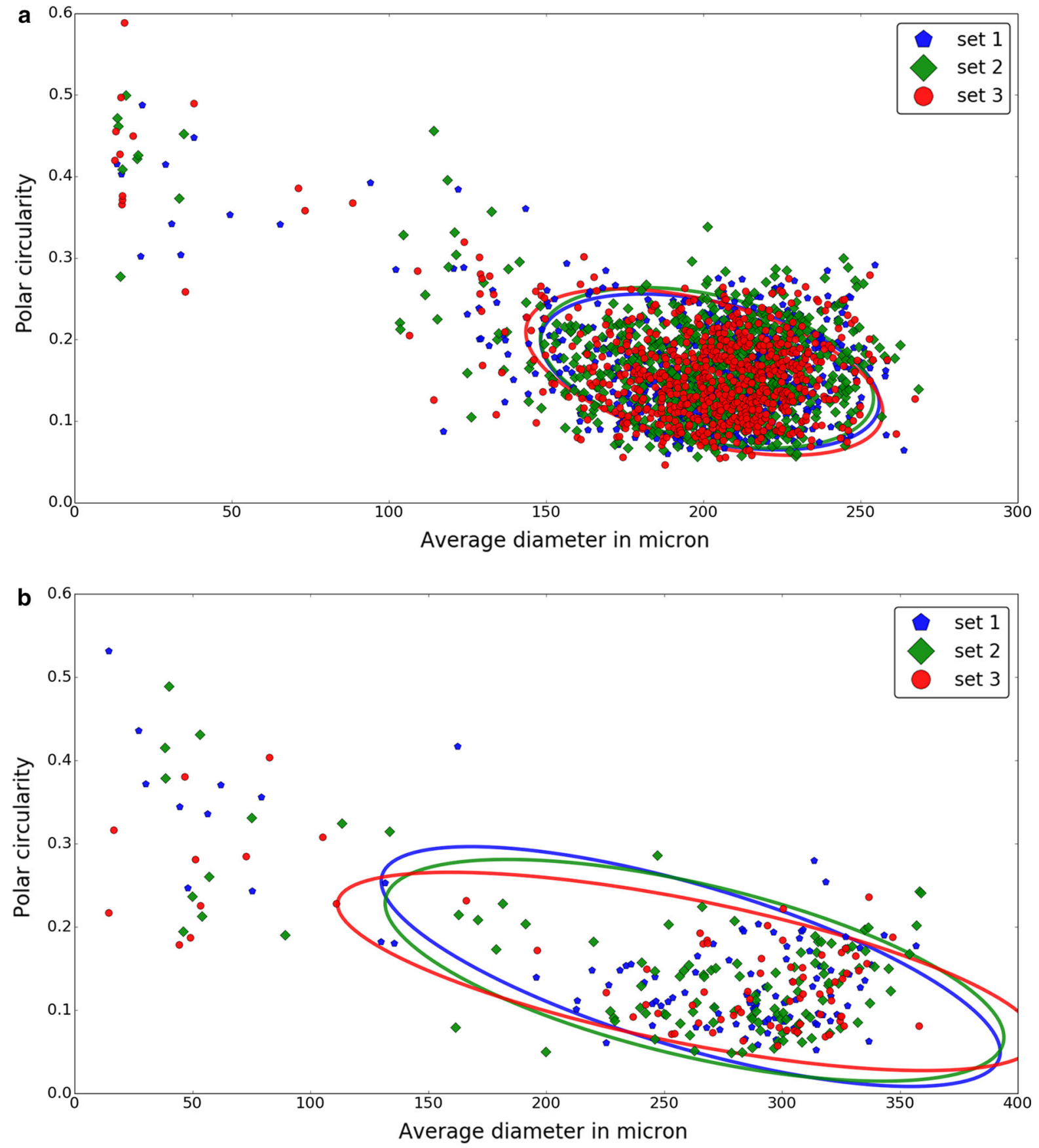

Fig. 2 Reproducibility of measurements performed with SParticle. a Analysis of particles from a submerged culture, performed in triplicate, revealed no significant differences in the average diameter versus the polar circularity between

circularity, no significant differences were found between the three replicate samples (Fig. 2a). This shows that the method reproducibly analyzed the replicates. The circles represent the $95 \%$ confidence intervals as determined with the error ellipse plotter (Kington 2012). b Likewise, no differences were observed when samples were taken after $24 \mathrm{~h}$ from different culture flasks

particles. Importantly, adding an identical inoculum to cultures grown in independent shake flasks also resulted in highly similar morphologies (Fig. 2b). 


\section{Correlating inoculum and pellet size}

To assess if we could distinguish between samples with a distinct pellet size distribution, cultures were inoculated at different spore densities. This was based on the observation that the inoculum density correlates to pellet size, where a higher number of spores results in a reduced average pellet size (Vecht-Lifshitz et al. 1990). For this, $25 \mathrm{ml}$ TSBS cultures were inoculated with $10^{5}, 10^{6}$ or $10^{7}$ cfu of $S$. coelicolor spores and samples were taken after 24 and $48 \mathrm{~h}$ of growth and analyzed by WSI. After $24 \mathrm{~h}$, no statistically relevant difference was observed between the three inoculation densities, which all yielded pellets with a similar diameter versus polar circularity (95\% CI; Fig. 3a). After $48 \mathrm{~h}$, small mycelial fragments represented a major fraction of all detected particles (Fig. 3b, left hand side). Fragmentation was observed at all inoculation densities, leading to the establishment of a population of small mycelial fragments and a separate population of larger particles. Notably, the average diameter of large particles was significantly different between the three cultures and depended on the starting inoculum (Fig. 3e), with $T$ test p-values of $1.1 \times 10^{-11}, 1.04 \times 10^{-74}, 1.46 \times 10^{-63}$ for $10^{5} \mathrm{vs}$. $10^{6}, 10^{5}$ vs. $10^{7}$ and $10^{6}$ vs. $10^{7}$, respectively. These differences between inoculum size and average pellet size, measured in the population from which small fragments were excluded, are in agreement with literature (Vecht-Lifshitz et al. 1990), which underlines the applicability of SParticle for the analysis of pellet morphologies.

\section{Influence of shaker speed and orbital size on pellet size}

Analysis of $24 \mathrm{~h}$ cultures at various shaker speeds and orbital sizes revealed no differences between baffled flasks or flasks with stainless steel spring coils (Fig. 4a). All other growth conditions were kept the same as described above. However, large differences were seen when flasks without baffles or springs were used (Fig. 4b). Decreasing the shaker speed from 200 to $100 \mathrm{rpm}$ led to a three-fold increase in average pellet diameter (Fig. 4c). We did not observe significant differences $(\mathrm{p}=0.09)$ between $125 \mathrm{ml}$ flasks and $250 \mathrm{ml}$ flasks filled with equal relative volumes (Fig. 4d), in line with previous observations (Mehmood et al. 2011).

\section{Application to other filamentous organisms}

To establish if SParticle can also be used to analyze the morphology of other filamentous microorganisms we analyzed the mycelia of $A$. niger $\mathrm{N} 402$ grown for $24 \mathrm{~h}$ and Gloeothece sp. PCC 6909, Chroococcidiopsis sp. PCC 6712 and S. elongatus PCC 7942 grown for $120 \mathrm{~h}$. From the image data, the software identified small and large pellets or assemblies of A. niger, Gloeothece sp. PCC 6909, Chroococcidiopsis sp. PCC 6712 and S. elongatus PCC 7942 (Fig. 5). This further underlines the applicability of SParticle for the analysis of the morphology of filamentous microorganisms in submerged cultures.

\section{Discussion}

Streptomyces species are exploited extensively as production hosts of natural products and enzymes. However, their complex morphology in submerged cultures such as during industrial fermentation significantly compromises their commercialization. Understanding the relationship between morphology and productivity of antibiotics and heterologous enzymes should, in part, identify how a strain may be morphologically tuned for optimal yield. Multiple methods have been applied to alter pellet morphology, based on changing environmental factors that alter growth or fragmentation (Mehmood et al. 2011; Wucherpfennig et al. 2011), on altering morphological genes such as those for surface polysaccharides [cslA, glxA, matAB (Chaplin et al. 2015; van Dissel et al. 2015; Xu et al. 2008)] or for cell division genes [ssgA (Traag and van Wezel 2008; van Wezel et al. 2006)]. Many of these morphological alterations have been shown to influence and often improve yield and productivity. This stresses the need for morphological optimization of Streptomyces cultures, where the relation between small changes in morphology and product formation is monitored. The new imaging tool developed during this work allows for detailed and at the same time rapid assessment of particle sizes and shapes in a given culture, thus enabling scientists to study the relationship between morphology and productivity efficiently and in high detail.

WSI-based image analysis represents a quick, easy and inexpensive method for analysis of pellet shapes and heterogeneity in Streptomyces cultures. The only 

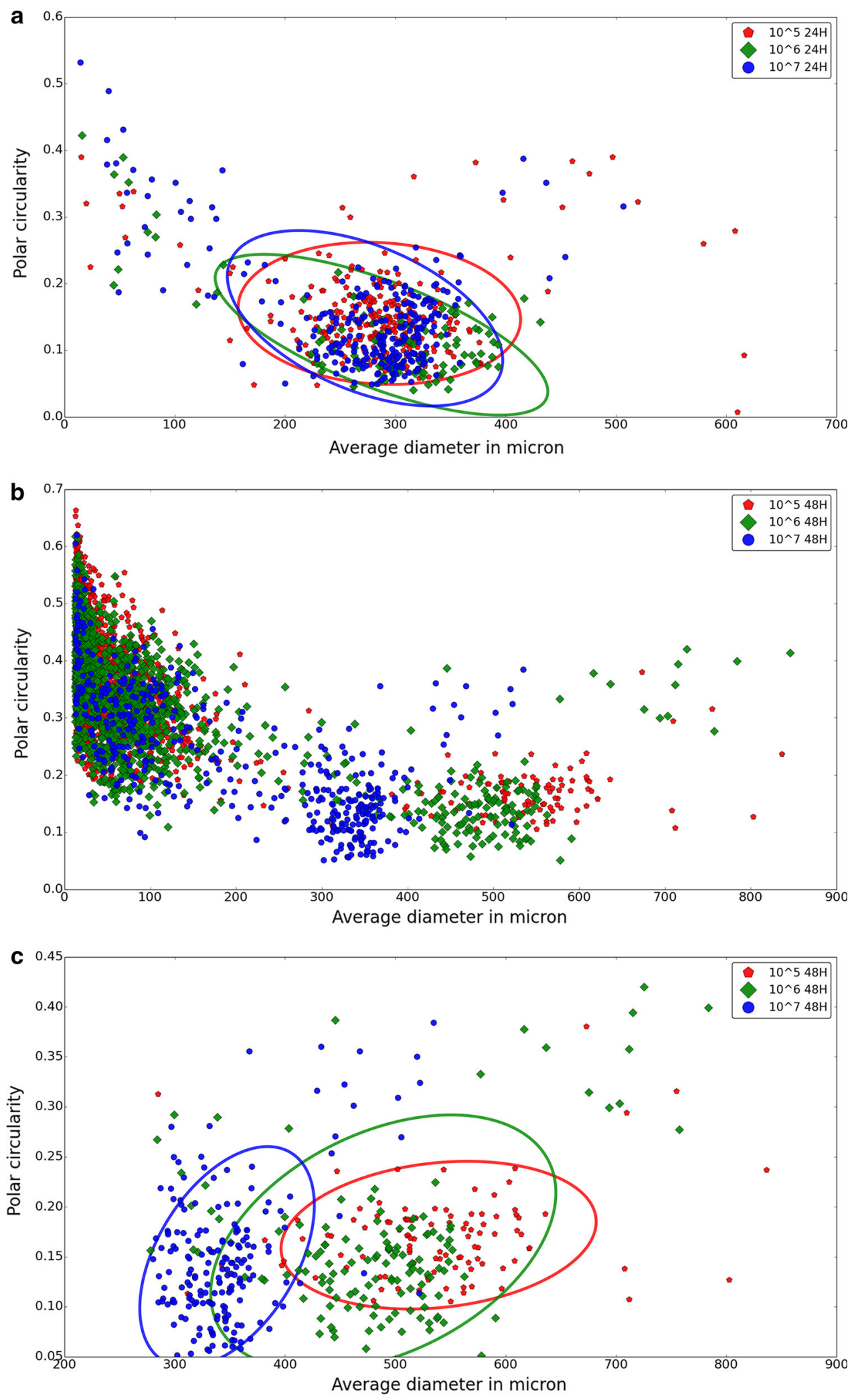
4Fig. 3 Correlation between inoculum and pellet morphology. a Inoculation density versus pellet size at $24 \mathrm{~h}$. Measurement on different inoculation densities after $24 \mathrm{~h}$ reveals no differences in pellet morphology in early growth. For increasing inoculation density the amounts of pellets analyzed were 276, 105, and 292 respectively. Plotted are the average diameter versus the polar circularity, whereby the circles represent the $95 \%$ confidence intervals. b Inoculation density versus pellet size at $48 \mathrm{~h}$. Measurements after $48 \mathrm{~h}$ indicate a large part of the population to be present as mycelial fragments and small pellets $(<150$ micron in length). The number of particles analyzed for increasing inoculation density were 1980, 1349, and 520 respectively. c Analysis of pellets from $48 \mathrm{~h}$ cultures confirms an inverse relationship between pellet size and inoculation density. The number of pellets analyzed for increasing inoculation density were 112, 149, and 213 respectively. Plotted are the average diameter versus the polar circularity; circles represent $95 \%$ confidence intervals

specialized equipment that is needed is an automated $\mathrm{XY}$-stage for a wide-field microscope. If such equipment, which is often routinely present in many imaging setups, is not available, the method can be used to automate the analysis of single images, although in that care must be taken to prevent subjective image selection. Besides the ease of
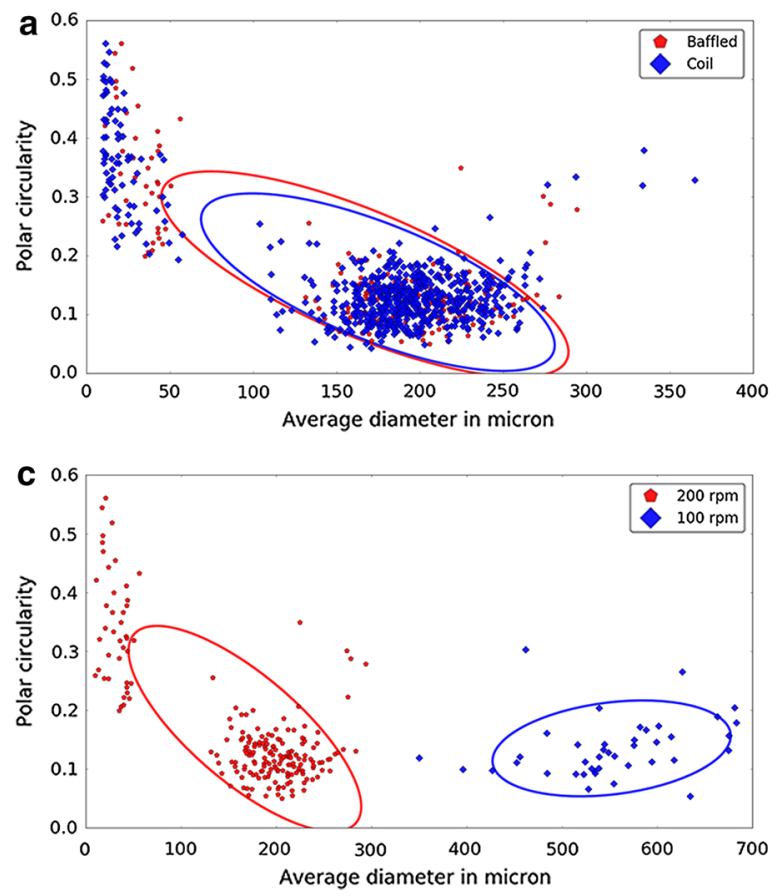

Fig. 4 Shake speed and orbital size versus pellet size. Differences between baffles, coils, common flasks, shake speeds and flask volume. Based on these data we see no differences between coils and baffles (a) or between different inoculation volumes (d). However clear differences in pellet size were
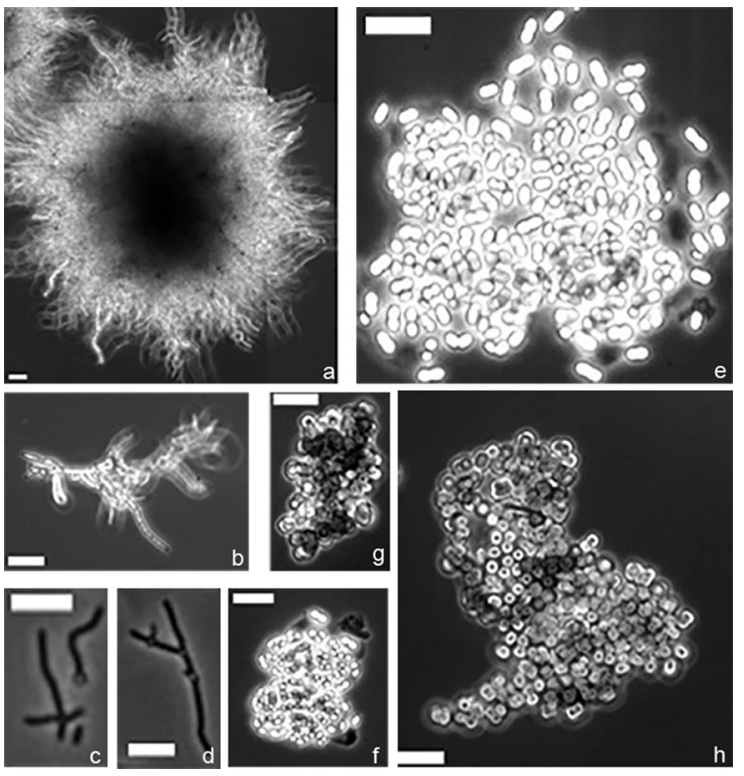

Fig. 5 Application of SParticle to image analysis for other filamentous organisms. Mycelial fragments and pellets were identified in cultures of Aspergillus niger (a, b), Synechococcus elongates PCC 7942 (c, d), Gloethece sp. PCC6909 (e, f), and Chroococcidiopsis sp. PCC $6712(\mathbf{g}, \mathbf{h})$. Scale bar, $5 \mu \mathrm{m}$
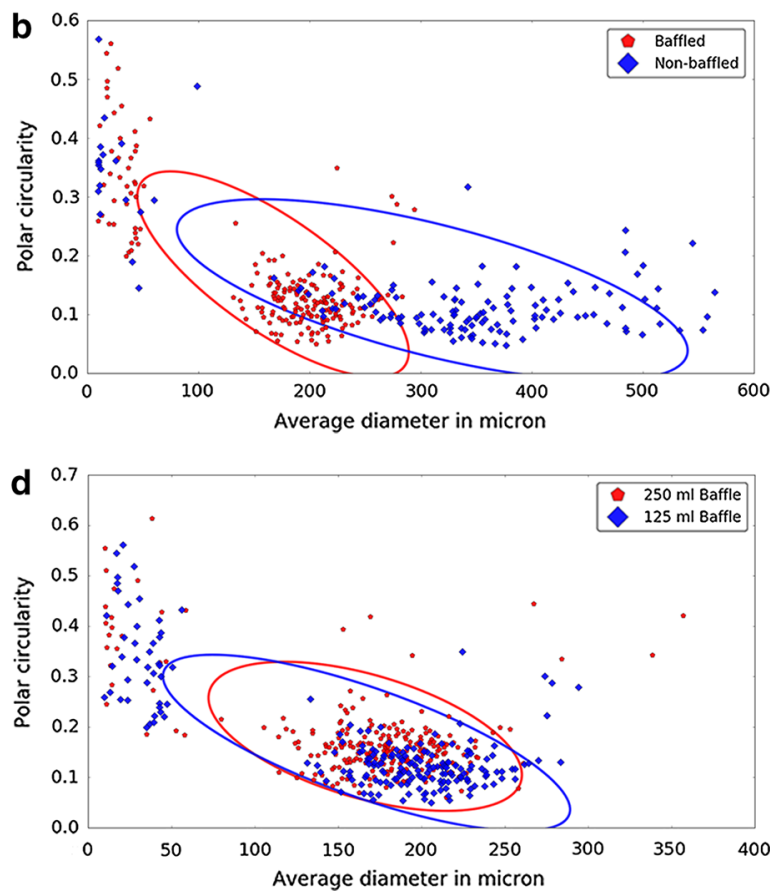

observed between unbaffled and baffled flasks (b) and when the orbital shaking speed was adjusted (c). Plotted are the average diameter versus the polar circularity; circles represent the $95 \%$ confidence intervals 
implementation, our method can also be adapted to include other microscopic methods, whereby qualitative pellet analysis may be based on for example fluorescence, such as for the study of mycelial aggregation (Zacchetti et al. 2016). In addition, the tool may be adapted for the analysis of filamentous bacteria in activated sludge processes where current tools are only $72 \%$ accurate (Dias et al. 2016).

Imaging mycelia formed after 24 and $48 \mathrm{~h}$ of growth with different inoculums were well in accordance with previously described dynamics. Imaging of a single slide containing up to 500 pellets and mycelial fragments can be done in $<10 \mathrm{~min}$. While this does not compare to the high rate of flow cytometry-based methods (COPAS), the throughput is high enough to analyze population dynamics and has the added advantage of being retraceable because the original images can be reanalyzed. Also, a COPAS is expensive, and the low-cost WSI setup makes the method widely applicable, and at much higher throughput than manual microscopic methods. Additional flexibility is offered by the fact that additional filters can be generated using the Roi_Manager_Filter_Creator tool. This allows creating a storable and reusable RoiManager filter allowing the user to set their own filtering parameters. Thus, the program can be adapted to analyze basically anything that can be separated from the background of given sample image by using intensity-based thresholding. The combination of tools should be widely applicable to anyone interested in automated image analysis of whole slide imaging.

Acknowledgements We are grateful to Gerben Voshol for providing Aspergillus niger N402, Gloeothece sp. PCC 6909, Chroococcidiopsis sp. PCC 6712 and Synechococcus elongatus PCC 7942.

Funding The work was supported by grants from the Netherlands Organization for Scientific Research (NWO VICI Grant 10379; VIDI Grant 12957).

Conflict of interest The authors declare that they have no conflict of interest.

Open Access This article is distributed under the terms of the Creative Commons Attribution 4.0 International License (http:// creativecommons.org/licenses/by/4.0/), which permits unrestricted use, distribution, and reproduction in any medium, provided you give appropriate credit to the original author(s) and the source, provide a link to the Creative Commons license, and indicate if changes were made.

\section{References}

Barka EA, Vatsa P, Sanchez L, Gavaut-Vaillant N, Jacquard C, Meier-Kolthoff J, Klenk HP, Clément C, Oudouch Y, van Wezel GP (2016) Taxonomy, physiology, and natural products of the Actinobacteria. Microbiol Mol Biol Rev 80:1-43

Barry DJ, Williams GA (2011) Microscopic characterisation of filamentous microbes: towards fully automated morphological quantification through image analysis. J Microsc 244:1-20

Bentley SD, Chater KF, Cerdeno-Tarraga AM, Challis GL, Thomson NR, James KD, Harris DE, Quail MA, Kieser H, Harper D, Bateman A, Brown S, Chandra G, Chen CW, Collins M, Cronin A, Fraser A, Goble A, Hidalgo J, Hornsby T, Howarth S, Huang CH, Kieser T, Larke L, Murphy L, Oliver K, O’Neil S, Rabbinowitsch E, Rajandream MA, Rutherford K, Rutter S, Seeger K, Saunders D, Sharp S, Squares R, Squares S, Taylor K, Warren T, Wietzorrek A, Woodward J, Barrell BG, Parkhill J, Hopwood DA (2002) Complete genome sequence of the model actinomycete Streptomyces coelicolor A3(2). Nature 417:141-147

Bérdy J (2005) Bioactive microbial metabolites. J Antibiot 58:1-26

Celler K, Picioreanu C, van Loosdrecht MC, van Wezel GP (2012) Structured morphological modeling as a framework for rational strain design of Streptomyces species. Antonie Van Leeuwenhoek 102:409-423

Chaplin AK, Petrus ML, Mangiameli G, Hough MA, Svistunenko DA, Nicholls P, Claessen D, Vijgenboom E, Worrall JA (2015) GlxA is a new structural member of the radical copper oxidase family and is required for glycan deposition at hyphal tips and morphogenesis of Streptomyces lividans. Biochem J 469:433-444

Chater KF, Losick R (1997) Mycelial life style of Streptomyces coelicolor A3(2) and its relatives. In: Shapiro JA, Dworkin $M$ (eds) Bacteria as multicellular organisms. Oxford University Press, New York, pp 149-182

Claessen D, Rozen DE, Kuipers OP, Sogaard-Andersen L, van Wezel GP (2014) Bacterial solutions to multicellularity: a tale of biofilms, filaments and fruiting bodies. Nat Rev Microbiol 12:115-124

Cooper LA, Kong J, Gutman DA, Dunn WD, Nalisnik M, Brat DJ (2015) Novel genotype-phenotype associations in human cancers enabled by advanced molecular platforms and computational analysis of whole slide images. Lab Invest 95:366-376

Cox PW, Thomas CR (1992) Classification and measurement of fungal pellets by automated image analysis. Biotechnol Bioeng 39:945-952

Cui YQ, Okkerse WJ, van der Lans RG, Luyben KC (1998) Modeling and measurements of fungal growth and morphology in submerged fermentations. Biotechnol Bioeng 60:216-229

Dias PA, Dunkel T, Fajado DA, Gallegos Ede L, Denecke M, Wiedemann P, Schneider FK, Suhr H (2016) Image processing for identification and quantification of filamentous bacteria in in situ acquired images. Biomed Eng Online $15: 64$ 
Girard G, Traag BA, Sangal V, Mascini N, Hoskisson PA, Goodfellow M, van Wezel GP (2013) A novel taxonomic marker that discriminates between morphologically complex actinomycetes. Open Biol 3:130073

Glazebrook MA, Doull JL, Stuttard C, Vining LC (1990) Sporulation of Streptomyces venezuelae in submerged cultures. J Gen Microbiol 136:581-588

Gray A, Wright A, Jackson P, Hale M, Treanor D (2015) Quantification of histochemical stains using whole slide imaging: development of a method and demonstration of its usefulness in laboratory quality control. J Clin Pathol 68:192-199

Hardy N, Moreaud M, Guillaume D, Augier F, Nienow A, Béal C, Ben Chaabane F (2017) Advanced digital image analysis method dedicated to the characterization of the morphology of filamentous fungus. J Microsc 266:126-140

Hopwood DA (2007) Streptomyces in nature and medicine: the antibiotic makers. Oxford University Press, New York

Jakimowicz D, van Wezel GP (2012) Cell division and DNA segregation in Streptomyces: how to build a septum in the middle of nowhere? Mol Microbiol 85:393-404

Kendrick KE, Ensign JC (1983) Sporulation of Streptomyces griseus in submerged culture. J Bacteriol 155:357-366

Kieser T, Bibb MJ, Buttner MJ, Chater KF, Hopwood DA (2000) Practical Streptomyces genetics. John Innes Foundation, Norwich

Kington JDI (2012) The structure and kinematics of the Nankai trough accretionary prism, Japan. University of Wisconsin-Madison, Madison

Mehmood N, Olmos E, Goergen JL, Blanchard F, Ullisch D, Klockner W, Buchs J, Delaunay S (2011) Oxygen supply controls the onset of pristinamycins production by Streptomyces pristinaespiralis in shaking flasks. Biotechnol Bioeng 108:2151-2161

Meyerhoff J, Tiller V, Bellgardt KH (1995) Two mathematical models for the development of a single microbial pellet. Biotechnol Bioeng 12:305-313

Obert M, Pfeifer P, Sernetz M (1990) Microbial growth patterns described by fractal geometry. J Bacteriol 172:1180-1185

Omura S (1992) Thom Award Lecture. Trends in the search for bioactive microbial metabolites. $\mathrm{J}$ Ind Microbiol 10:135-156

Packer HL, Thomas CR (1990) Morphological measurements on filamentous microorganisms by fully automatic image analysis. Biotechnol Bioeng 35:870-881

Papagianni M (2014) Characterization of fungal morphology using digital image analysis techniques. J Microb Biochem Technol 6:189-194

Petrus ML, van Veluw GJ, Wösten HA, Claessen D (2014) Sorting of Streptomyces cell pellets using a complex object parametric analyzer and sorter. J Vis Exp. doi:10.3791/ 51178

Pinto LS, Vieira LM, Pons MN, Fonseca MM, Menezes JC (2004) Morphology and viability analysis of Streptomyces clavuligerus in industrial cultivation systems. Bioprocess Biosyst Eng 26:177-184

Posch AE, Spadiut O, Herwig C (2012) A novel method for fast and statistically verified morphological characterization of filamentous fungi. Fungal Genet Biol 49:499-510
Reichl U, Buschulte TK, Gilles ED (1990) Study of the early growth and branching of Streptomyces tendae by means of an image processing system. J Microsc 158:55-62

Reichl U, King R, Gilles ED (1992) Characterization of pellet morphology during submerged growth of Streptomyces tendae by image analysis. Biotechnol Bioeng 39:164-170

Rønnest NP, Stocks SM, Lantz AE, Gernaey KV (2012) Comparison of laser diffraction and image analysis for measurement of Streptomyces coelicolor cell clumps and pellets. Biotechnol Lett 34:1465-1473

Ryoo D (1999) Fungal fractal morphology of pellet formation in Aspergillus niger. Biotechnol Tech 13:33-36

Stojmenovic M, Jevremovic A, Nayak A (2013) Fast iris detection via shape based circularity. In: 2013 8th IEEE conference on industrial electronics and applications (ICIEA), 19-21 June 2013, pp 747-752

Tough AJ, Prosser JI (1996) Experimental verification of a mathematical model for pelleted growth of Streptomyces coelicolor A3(2) in submerged batch culture (vol 142, pg 639, 1996). Microbiology 142:1332

Traag BA, van Wezel GP (2008) The SsgA-like proteins in actinomycetes: small proteins up to a big task. Antonie Van Leeuwenhoek 94:85-97

Treskatis SK, Orgeldinger V, Wolf H, Gilles ED (1997) Morphological characterization of filamentous microorganisms in submerged cultures by on-line digital image analysis and pattern recognition. Biotechnol Bioeng 53:191-201

Tresner HD, Hayes JA, Backus EJ (1967) Morphology of submerged growth of Streptomycetes as a taxonomic aid. I. Morphological development of Streptomyces aureofaciens in agitated liquid media. Appl Microbiol 15:1185

van Dissel D, Claessen D, Van Wezel GP (2014) Morphogenesis of Streptomyces in submerged cultures. Adv Appl Microbiol 89:1-45

van Dissel D, Claessen D, Roth M, van Wezel GP (2015) A novel locus for mycelial aggregation forms a gateway to improved Streptomyces cell factories. Microb Cell Fact $14: 44$

van Wezel GP, Krabben P, Traag BA, Keijser BJ, Kerste R, Vijgenboom E, Heijnen JJ, Kraal B (2006) Unlocking Streptomyces spp. for use as sustainable industrial production platforms by morphological engineering. Appl Environ Microbiol 72:5283-5288

van Wezel GP, McKenzie NL, Nodwell JR (2009) Chapter 5. Applying the genetics of secondary metabolism in model actinomycetes to the discovery of new antibiotics. Methods Enzymol 458:117-141

Vecht-Lifshitz SE, Magdassi S, Braun S (1990) Pellet formation and cellular aggregation in Streptomyces tendae. Biotechnol Bioeng 35:890-896

Vinck A, Terlou M, Pestman WR, Martens EP, Ram AF, van den Hondel CA, Wosten HA (2005) Hyphal differentiation in the exploring mycelium of Aspergillus niger. Mol Microbiol 58:693-699

Voshol GP, Meyer V, van den Hondel CA (2015) GTP-binding protein Era: a novel gene target for biofuel production. BMC Biotechnol 15:21

Wardell JN, Stocks SM, Thomas CR, Bushell ME (2002) Decreasing the hyphal branching rate of Saccharopolyspora erythraea NRRL 2338 leads to increased 
resistance to breakage and increased antibiotic production. Biotechnol Bioeng 78:141-146

Webster JD, Dunstan RW (2014) Whole-slide imaging and automated image analysis: considerations and opportunities in the practice of pathology. Vet Pathol 51:211-223

Willemse J, van Wezel GP (2009) Imaging of Streptomyces coelicolor A3(2) with reduced autofluorescence reveals a novel stage of FtsZ localization. PLoS ONE 4:e4242

Willemse J, Ruban-Osmialowska B, Widdick D, Celler K, Hutchings MI, van Wezel GP, Palmer T (2012) Dynamic localization of Tat protein transport machinery components in Streptomyces coelicolor. J Bacteriol 194:6272-6281

Wucherpfennig T, Hestler T, Krull R (2011) Morphology engineering —osmolality and its effect on Aspergillus niger morphology and productivity. Microb Cell Fact 10:58
Wucherpfennig T, Lakowitz A, Krull R (2013) Comprehension of viscous morphology-evaluation of fractal and conventional parameters for rheological characterization of Aspergillus niger culture broth. J Biotechnol 163:124-132

Xu H, Chater KF, Deng Z, Tao M (2008) A cellulose synthaselike protein involved in hyphal tip growth and morphological differentiation in Streptomyces. J Bacteriol 190:4971-4978

Zacchetti B, Willemse J, Recter B, van Dissel D, van Wezel GP, Wosten HA, Claessen D (2016) Aggregation of germlings is a major contributing factor towards mycelial heterogeneity of Streptomyces. Sci Rep 6:27045 